\title{
Role of Melatonin in Arbuscular Mycorrhizal Fungi-Induced Resistance to Fusarium Wilt in Cucumber
}

\author{
Golam Jalal Ahammed, ${ }^{\dagger}$ Qi Mao, Yaru Yan, Meijuan Wu, Yaqi Wang, Jingjing Ren, Pan Guo, Airong Liu, and \\ Shuangchen Chen ${ }^{\dagger}$
}

College of Forestry, Henan University of Science and Technology, Luoyang, 471023, P.R. China

Accepted for publication 22 February 2020.

ABSTRACT

\begin{abstract}
Melatonin is a multifunctional molecule that confers tolerance to a number of biotic and abiotic stresses in plants. However, the role of melatonin in plant response to Fusarium oxysporum and the interaction with arbuscular mycorrhizal fungi (AMF) remain unclear. Here we show that exogenous melatonin application promoted the AMF colonization rate in cucumber roots, which potentially suppressed Fusarium wilt as evidenced by a decreased disease index and an increased control effect. Leaf gas exchange analysis revealed that Fusarium inoculation significantly decreased the net photosynthetic rate (Pn), stomatal conductance $(\mathrm{Gs})$, intercellular $\mathrm{CO}_{2}$ concentrations $(\mathrm{Ci})$, and transpiration rate $(\mathrm{Tr})$. Intriguingly, either melatonin application or AMF inoculation significantly increased the $\mathrm{Pn}, \mathrm{Gs}$, Tr, and dry biomass, and their combined treatment showed a more profound effect under Fusarium stress. Further analysis showed that Fusarium induced oxidative stress as evidenced by increased lipid peroxidation and electrolyte leakage. Conversely, either
\end{abstract}

melatonin or AMF drastically attenuated the levels of malondialdehyde, $\mathrm{H}_{2} \mathrm{O}_{2}$, and electrolyte leakage in Fusarium-inoculated plants, and their combined treatment caused a further decrease. Fusarium inoculation decreased the activity and transcripts of superoxide dismutase and ascorbate peroxidase, and the content of glutathione and proline. Besides, the activity and transcripts of peroxidase and catalase, the content of phenols and flavonoids increased after Fusarium infection. Importantly, melatonin and/or AMF significantly increased those parameters with the greatest effect with their combined treatment under Fusarium stress. Our results suggest that a positive collaboration between melatonin and AMF enhances resistance to Fusarium wilt in cucumber plants.

Keywords: antioxidants, arbuscular mycorrhizal fungi, biochemistry and cell biology, biological control, disease control and pest management, disease resistance, Fusarium wilt, melatonin, photosynthesis
Plant pathogenic fungi are one of the major biotic hazards that negatively affect plant growth and productivity around the world. Fusarium oxysporum is a filamentous plant pathogenic fungus that causes wilt disease in a number of important crops (Gordon 2017). Fusarium colonization clogs the vascular system, leading to severe wilting of aerial plant parts (Zhang et al. 2016). To control Fusarium wilt, a large amount of fungicides are irrationally used all over the world, leading to nontargeted adverse effects on the environment (Gordon 2017). Therefore, the development of efficient and environmentally friendly wilt management strategies has been a key research focus in recent years (Chen et al. 2019). These include enhancement of plant resistance by symbiotic association with beneficial fungi, such as Trichoderma spp., as well as

${ }^{\dagger}$ Corresponding authors: S. Chen; chen_shuangchen@126.com, and G. Jalal Ahammed; ahammed@haust.edu.cn

Author contributions: Conceived and designed the experiments: G.J.A. and S.C.; performed the experiments: M.W., Y.W., Y.Y., P.G., and G.J.A.; analyzed the data: G.J.A., S.C., Q.M., J.R., and A.L.; wrote the draft manuscript: G.J.A.; and reviewed and edited the manuscript: G.J.A. and S.C. All authors have read and approved the manuscript.

Funding: This project was funded by the National Key Research and Development Program of China (2018YFD1000800), the National Natural Science Foundation of China (31872092, 31872157, and 31950410555), Program for Science and Technology Innovation Talents in Universities of Henan Province (19HASTIT009), Henan Natural Science Foundation (182300410046, 182300410090), Henan University of Science and Technology Research Start-up Fund for New Faculty (13480058), and the Key Laboratory of Horticultural Crop Growth and Quality Control in Protected Environment of Luoyang City.

*The $\boldsymbol{e}$-Xtra logo stands for "electronic extra" and indicates that one supplementary table is published online.

The author(s) declare no conflict of interest.

(c) 2020 The American Phytopathological Society pharmacological treatments with plant growth regulators or signaling molecules that can increase plant defense against fungal pathogens (Chen et al. 2019; Li et al. 2016).

Arbuscular mycorrhizal fungi (AMF) are soilborne beneficial fungi that make symbiotic associations with plant roots and provide a variety of benefits to the host plants (Chen et al. 2017b; Shi et al. 2013, 2017). Moreover, AMF can enhance plant tolerance to many biotic and abiotic stresses and thus they can be used to control fungal diseases (Aseel et al. 2019; Bahadur et al. 2019; Li et al. 2019c; Mathur et al. 2018; Miozzi et al. 2019; Oyewole et al. 2017; Rathod et al. 2011). For instance, inoculation with Funneliformis mosseae enhances tolerance to Phoma medicaginis, the causal agent of leaf spots in alfalfa (Medicago sativa), and combined inoculation with Glomus intraradices, Glomus claroideum, and Aphanomyces euteiches suppresses root rot in pea (Pisum sativum) (Gao et al. 2018; Li et al. 2019c). Importantly, AMF-enhanced tolerance to leaf spot disease is attributed to the increased activity of antioxidant enzymes (Gao et al. 2018; Li et al. 2019c). Moreover, it is believed that AMF-enhanced tolerance to plant pathogens is also associated with the stimulation of plant secondary metabolism. For instance, mycorrhizal colonization with Rhizophagus intraradices decreases leaf spot disease index by $56.18 \%$ in alfalfa, which is associated with differential expression of genes involved in phenylpropanoid biosynthesis, flavonoid biosynthesis, glutathione (GSH) metabolism, and so on ( $\mathrm{Li}$ et al. 2019c). Moreover, AMF colonization increases resistance to Tomato mosaic virus by enhancing the expression of PHENYLALANINE AMMONIA-LYASE 1 (PAL1) and the content of flavonoids in tomato (Aseel et al. 2019).

Photosynthesis is the most vital physiological process in plants. But infections with disease causal pathogens negatively affect plant photosynthetic capacity, leading to significant yield losses (Sun et al. 2019). RNA-seq analysis revealed that infection with Phoma medicaginis down-regulates a number of genes in the photosynthetic pathway, including a gene encoding for the photosystem 1 
reaction center subunit $\mathrm{N}$, suggesting the negative effects of fungal pathogens on photosynthesis ( $\mathrm{Li}$ et al. 2019c). Notably, enzymes involved in the photosynthetic light reaction, as well as the components of photosystem I and II, are sensitive to reactive oxygen species (ROS) (Ahammed et al. 2018; Sun et al. 2019). It is well established that fungal infections increase ROS accumulation, which can damage biological membranes, such as thylakoid membranes (Chen et al. 2019; Zhang et al. 2016). To ameliorate ROS-caused oxidative stress, plants elevate the activities of antioxidant enzymes (Ahammed et al. 2019). In addition to the enzymatic antioxidants, the nonenzymatic antioxidants, such as flavonoids that are synthesized through the secondary metabolic pathway, are capable of scavenging ROS (Aseel et al. 2019). Thus, enhancement of plant antioxidant defense and secondary metabolism can strengthen plant defense against plant pathogenic fungi.

Melatonin ( $N$-acetyl-5-methoxytryptamine), a multifunctional biomolecule in plants, can stimulate photosynthetic processes and growth under biotic and abiotic stresses (Ahammed et al. 2019, 2020; Hasan et al. 2019; Hernández-Ruiz and Arnao 2018; Li et al. 2018, 2019a; Sharma and Zheng 2019). Classically being a strong antioxidant and an inducer of plant stress tolerance, melatonin can enhance plant resistance to a number of fungal diseases, such as gray mold in cherry tomato caused by Botrytis cinerea (Li et al. 2019a), anthracnose in banana caused by Colletotrichum musae ( $\mathrm{Li}$ et al. 2019b), tomato fruit decay caused by Botrytis cinerea (Liu et al. 2019), powdery mildew in watermelon caused by Podosphaera xanthii and crown rot in cucurbits caused by soilborne fungus Phytophthora capsici (Mandal et al. 2018), downy mildew in cucumber caused by Pseudoperonospora cubensis (Sun et al. 2019), and potato late blight caused by Phytophthora infestans (Zhang et al. 2017). In cucumber, melatonin application decreased the levels of lipid peroxidation and relative electrolyte leakage caused by Pseudoperonospora cubensis, which was associated with the increased activity of antioxidant enzymes in leaves (Sun et al. 2019). However, melatonin treatment enhances susceptibility to Penicillium digitatum in citrus fruits by attenuating the content of $\mathrm{H}_{2} \mathrm{O}_{2}$, suggesting a negative role for melatonin in defense against fungal pathogens that cause postharvest diseases in citrus (Lin et al. 2019). Despite this fact, both melatonin and AMF are considered effective stimulators of plant defense against fungal pathogens (Hernández-Ruiz and Arnao 2018); however, the role of melatonin in AMF-induced resistance to Fusarium oxysporum remains unclear. In this study, we unraveled an additive role of melatonin and AMF that could enhance cucumber resistance to Fusarium wilt to a greater extent than their sole treatment. Our study unveiled an eco-friendly wilt management strategy by combining the application of melatonin and AMF in cucumber plants.

\section{MATERIALS AND METHODS}

Plant culture, fungal inoculation and treatments. $\mathrm{Cu}-$ cumber (Cucumis sativus L. 'Jinyou 28') seeds were surface sterilized with a solution of sodium hypochlorite $(5 \%, \mathrm{vol} / \mathrm{vol})$ for $10 \mathrm{~min}$ followed by rinsing with distilled water four times. Then the seeds were germinated on layers of wet filter paper at $28^{\circ} \mathrm{C}$. When the seeds germinated (after 4 days of germination treatment), they were transplanted in plastic pots $(13 \times 13 \mathrm{~cm})$ containing sterilized soil substrate $(0.88 \mathrm{~kg}$; organic manure/soil/decomposed straw $=1$ : $2: 1$ ) with the composition described previously (Chen et al. 2013). During transplanting, seedlings under the AMF treatments (AMF plants) were inoculated with AMF by adding $10 \mathrm{~g}$ of Glomus claroideum per pot containing approximately 720 spores. The AMF inocula contained spores, soil, extraradical hyphae, and infected clove (Trifolium repens L.) root fragments from a stock culture of G. claroideum. The AMF inocula were placed close to the roots of seedlings (Chen et al. 2013). Treatments not receiving the AMF (non-AMF plants) were inoculated with the same amount of sterilized (autoclaved) AMF inocula. From the fifth day after transplanting, the foliage of seedlings under melatonin treatment was sprayed with $100 \mu \mathrm{M}$ melatonin (Sigma-Aldrich, supplier Mainland, China) in the dark at 5-day intervals. Melatonin solution was prepared by dissolving the melatonin in a small amount of ethanol followed by dilution at a 1:10,000 ratio with double distilled water (ethanol/ $\mathrm{ddH}_{2} \mathrm{O}, \mathrm{vol} / \mathrm{vol}$ ). Treatments that did not receive melatonin were sprayed with $\mathrm{ddH}_{2} \mathrm{O}$ containing the same ratio of ethanol. Twenty days after transplanting, biotic stress was imposed by inoculating plants with Fusarium oxysporum f. sp. cucumerinum at $1 \times 10^{6} \mathrm{CFU} / \mathrm{ml}$ (20 ml per plant) via soil drenching. Thus, the study consisted of eight treatments, such as (i) control (sprayed with distilled water and received only autoclaved inocula), (ii) AMF (sprayed with distilled water and inoculated with active AMF inocula), (iii) MT (sprayed with $100 \mu \mathrm{M}$ melatonin and inoculated with autoclaved AMF inocula), (iv) AMF+MT (sprayed with $100 \mu \mathrm{M}$ melatonin and inoculated with active AMF inocula), (v) FO (sprayed with distilled water and inoculated with spores of Fusarium oxysporum), (vi) AMF+FO (sprayed with distilled water and inoculated with both AMF and Fusarium oxysporum), (vii) $\mathrm{MT}+\mathrm{FO}$ (sprayed with $100 \mu \mathrm{M}$ melatonin and inoculated with Fusarium oxysporum), and (viii) $\mathrm{AMF}+\mathrm{MT}+\mathrm{FO}$ (sprayed with $100 \mu \mathrm{M}$ melatonin and inoculated with both AMF and Fusarium oxysporum). The methods for the preparation of AMF and Fusarium oxysporum inocula were described previously (Chen et al. 2013, 2019). The experimental layout was a completely randomized design, with eight treatments and three replicates for each treatment, whereas each replicate contained six cucumber plants. The present study was carried out in a solar greenhouse and the average temperatures were $28^{\circ} \mathrm{C} / 20^{\circ} \mathrm{C}$ (day/night) during the experimental period with a photosynthetic photon flux density (PPFD) of $600 \mu \mathrm{mol} \mathrm{m}{ }^{-2} \mathrm{~s}^{-1}$ and relative humidity of $80 \%$. Fifteen days after the inoculation with Fusarium oxysporum, six cucumber plants per treatment were randomly harvested and kept in an oven at $80^{\circ} \mathrm{C}$ for $72 \mathrm{~h}$ to measure the dry weights. Eventually, the experiment was terminated 15 days after the inoculation with Fusarium oxysporum by measuring the gas exchanging parameters followed by the collection of leaf/root samples (in case of leaf samples, the second fully expanded leaf was collected from each plant) for different analyses. The samples were immediately frozen in liquid nitrogen (unless otherwise stated) and stored at $-80^{\circ} \mathrm{C}$ until further analyses.

Determination of mycorrhizal colonization rate, disease index, and control effect. The gridline intercept method was used to determine the mycorrhizal colonization rate on 35 days after inoculation with AMF as previously described (Chen et al. 2013; Giovannetti and Mosse 1980). Disease index was analyzed based on the indexation of root rot and vascular bundle browning, representing different disease rating scores as follows: healthy roots without any browning were given a value of 0 , white roots with scarce browning (less than 5\% of total root) were given a value of 1 , roots with slight rot and browning ( 5 to $20 \%$ of total root) received a value of 2, mild root rot and browning (above 20 to $50 \%$ of total root) received a value of 3 , and roots with severe rot and browning (above 50 to $100 \%$ of total root) were given a value of 4 (Chen et al. 2017a; Ding et al. 2014). Disease index (DI) was calculated according to the following formula: $\mathrm{DI}=\left[\sum\right.$ (rating score $\times$ number of plants rated)/(total number of plants $\times$ highest rating score $) \times$ 100]. Subsequently, the control effect was estimated based on DI as follows: Control effect $(\%)=[($ control DI - treatment DI $) /$ control DI] $\times 100$ (Chen et al. 2019).

Determination of gas exchange parameters in cucumber leaves. Fifteen days after the inoculation with Fusarium oxysporum, leaf gas exchange was measured in each of the second fully expanded leaf of six cucumber plants for each treatment between 8 : 00 a.m. and 11:00 a.m. Data of gas exchange parameters, such as net $\mathrm{CO}_{2}$ assimilation rate $(\mathrm{Pn})$, intercellular $\mathrm{CO}_{2}$ concentration $(\mathrm{Ci})$, stomatal conductance $(\mathrm{Gs})$, and transpiration rate (Tr) were generated by a LI-6400 Portable Photosynthesis System, an openflow infrared gas analyzer adapted with light and temperature 
control systems (LI-6400; LICOR, Lincoln, NE, U.S.A.) for each leaf sample. The following conditions were maintained during gas exchange assay: $25^{\circ} \mathrm{C}$ of air temperature, 80 to $90 \%$ relative humidity, $400 \mu \mathrm{mol} \mathrm{mol}-1$ of $\mathrm{CO}_{2}$ concentration, and 1,000 $\mu \mathrm{mol}$ $\mathrm{m}^{-2}$ PPFD.

Determination of hydrogen peroxide, lipid peroxidation, cell viability, and electrolyte leakage. For the assay of hydrogen peroxide $\left(\mathrm{H}_{2} \mathrm{O}_{2}\right)$ and malondialdehyde (MDA) concentrations, $0.5 \mathrm{~g}$ of leaf tissue was used as each sample unit collected from the second fully expanded leaf of each plant and pooled in triplicate from six plants. $\mathrm{H}_{2} \mathrm{O}_{2}$ content was assayed spectrophotometrically through a peroxidase assay as described by Willekens et al. (1997). In situ $\mathrm{H}_{2} \mathrm{O}_{2}$ accumulation was visualized through the histochemical staining of leaves with 3,3-diaminobenzidine (DAB) as previously described (Chen et al. 2013). The second fully expanded cucumber leaves of six plants for each treatment were used for DAB staining. MDA content in leaves was assayed as a marker of lipid peroxidation following the thiobarbituric acid (TBA) reaction as described elsewhere (Ahammed et al. 2019). Briefly, leaf tissues $(0.5 \mathrm{~g})$ were extracted in potassium phosphate buffer $(50 \mathrm{mM})$. The homogenates were centrifuged for $20 \mathrm{~min}$ at $12,000 \times g$. Then, $4 \mathrm{ml}$ of $0.65 \%$ TBA in $20 \%$ TCA (trichloroacetic acid) solution was mixed and the mixture was heated in a hot water bath at $95^{\circ} \mathrm{C}$ for $30 \mathrm{~min}$. Afterward, the tubes were quickly placed in an ice bucket to end the reaction. The samples were then centrifuged for $5 \mathrm{~min}$ at $10,000 \times \mathrm{g}$, and the absorbance of the red adduct was measured at 440, 532, and $600 \mathrm{~nm}$ with a spectrophotometer (SHIMADZU UV-2410PC, Shimadzu Co., Kyoto, Japan). MDA equivalents were quantified and expressed as MDA content per gram fresh weight (Ahammed et al. 2019). To assess the root cell viability, root tips (six root tips per treatment) were stained with fluorescein diacetate (FDA) and propidium iodide (PI), and the specific fluorescence from the stained roots was observed under a fluorescent microscope (Zeiss LSM510) as described previously (Chen et al. 2017a). For the assay of root activity, $0.5 \mathrm{~g}$ of fresh root sample was used as each sample unit in triplicate. Root samples were a mixture of samples from the six plants per treatment, which were cleaned with water and wiped with tissue paper before weighing. The root activity was determined according to the triphenyl tetrazolium chloride (TTC) method as previously described (Chen et al. 2017b). For the assay of electrolyte leakage (\%), $0.3 \mathrm{~g}$ of fresh leaf sample of equal size was harvested as each sample unit in triplicate from different plants. Electrolyte leakage (\%) was analyzed by measuring the electrical conductivity (EC) of leaf suspension. The first reading (EC1) was taken before autoclaving, whereas the second reading (EC2) was taken after autoclaving (Ahammed et al. 2019). The electrolyte leakage percentage was determined according to the following formula: Electrolyte leakage $(\%)=100 \times \mathrm{EC} 1 / \mathrm{EC} 2$.

Determination of antioxidant enzyme activity. To extract antioxidant enzymes, frozen leaf tissues ( $0.3 \mathrm{~g}$ sample in triplicate) were crushed with ice-cold HEPES buffer $(3 \mathrm{ml}, 25 \mathrm{mM}$, and $\mathrm{pH}$ $7.8)$ containing EDTA $(0.2 \mathrm{mM})$ and insoluble polyvinylpyrrolidone $(2 \%, \mathrm{wt} / \mathrm{vol})$. The buffer was augmented with ascorbic acid $(2 \mathrm{mM})$ for the extraction of ascorbate peroxidase (APX) only. The homogenates were centrifuged at $4^{\circ} \mathrm{C}$ for $20 \mathrm{~min}$ at $12,000 \times g$. The supernatants were used for the analysis of the activity of antioxidant enzymes. The activity of superoxide dismutase (SOD) was analyzed based on its capacity to inhibit the photoreduction of nitro blue tetrazolium chloride (Giannopolitis and Ries 1977). The activity of catalase (CAT) and peroxidase (POD) was determined as described previously (Cakmak and Marschner 1992). The activity of APX was analyzed based on the $\mathrm{H}_{2} \mathrm{O}_{2}$-dependent oxidation of ascorbic acid as described previously (Nakano and Asada 1981). The activity of antioxidant enzymes was presented on the basis of protein content, whereas the protein content was assessed using bovine serum albumin as the standard as described previously (Bradford 1976).
Assay of antioxidant and secondary metabolite content. For the assay of GSH content, leaf samples ( $0.2 \mathrm{~g}$ sample in triplicate) were homogenized in $6 \%$ metaphosphoric acid ( $\mathrm{pH} 2.8)$, and the homogenates were centrifuged for $15 \mathrm{~min}$ at $12,000 \times \mathrm{g}$. Then one half of the supernatant was used for the assay of the total glutathione (GSH + GSSG), while the other half was used for the quantification of the oxidized glutathione (GSSG). Notably, incubation of the supernatants for an hour with 2-vinylpyridine eventually blocked the reduced glutathione (GSH). Then following reagents such as $100 \mathrm{mM}$ phosphate buffered saline $(\mathrm{pH} 7.5), 5 \mathrm{mM}$ EDTA, $0.2 \mathrm{mM}$ NADPH, $0.6 \mathrm{mM}$ DTNB, and $3 \mathrm{U}$ of glutathione reductase from yeast (Sigma, Santa Clara, CA, U.S.A.) were mixed to initiate the reaction and the absorbance was measured spectrophotometrically at $412 \mathrm{~nm}$ for $1 \mathrm{~min}$. The GSH content was calculated by deducting the content of GSSG from total GSH (Rahman et al. 2006).

Proline content was measured based on sulfosalicylic acid colorimetry. In brief, leaf samples $(0.5 \mathrm{~g}$ sample in triplicate $)$ were homogenized in a 10-ml centrifuge tube, followed by the addition of $3 \%$ aqueous sulfosalicylic acid $(5 \mathrm{ml})$. After keeping the tubes in boiling water for $10 \mathrm{~min}$, an aliquot of the supernatant $(2 \mathrm{ml})$ was mixed to initiate the reaction with the freshly prepared acidic ninhydrin $(2 \mathrm{ml})$ and glacial acetic acid $(2 \mathrm{ml})$ in a test tube and kept at $100^{\circ} \mathrm{C}$ for $30 \mathrm{~min}$. Afterward, the tubes were immediately placed in an ice bath to terminate the reaction, and the mixture was then extracted with toluene $(4 \mathrm{ml})$ and vortexed vigorously for $20 \mathrm{~s}$. Then the chromophore-containing toluene was aspirated from the aqueous phase, and the absorbance was determined photometrically at $520 \mathrm{~nm}$ using toluene for a blank (Zhang et al. 2019). For the determination of total phenol content, a method based on FolinCiocalteu's reagent reduction was used (Ahammed et al. 2020). Total flavonoid concentration was analyzed spectrophotometrically as previously described. In brief, leaf samples were extracted in $70 \%$ ethanol (vol/vol, ethanol/water) at $100^{\circ} \mathrm{C}$, and the absorbance of aqueous extract was recorded at $510 \mathrm{~nm}$ using $\mathrm{AlCl}_{3}$ method. Rutin was used as the standard for the calculation of total flavonoid content (Chen et al. 2013).

Total RNA extraction and qRT-PCR assay. Total RNA was isolated from $\sim 100 \mathrm{mg}$ leaf sample in three biological replicates using a total RNA extraction kit according to the manufacturer's instruction (Tiangen, Shanghai, China). An aliquot ( $1 \mu \mathrm{g})$ of total RNA was subjected to reverse-transcription to generate cDNA using specific kits (ReverTra Ace qPCR RT Kit, Toyobo, Japan). For qRTPCR assay, we used the SYBR Green PCR Master Mix (Takara, Tokyo, Japan). The qRT-PCR assay was performed with LightCycler 480 II Real-Time PCR Detection System (Roche, Basel, Swiss) under the default thermal cycling conditions with an added melting curve. The gene-specific primer pairs were designed for each gene and the actin was used as an internal control (Supplementary Table S1). The PCR was run at the following conditions: $95^{\circ} \mathrm{C}$ for $3 \mathrm{~min}$, followed by 40 cycles at $95^{\circ} \mathrm{C}$ for $30 \mathrm{~s}, 58^{\circ} \mathrm{C}$ for $30 \mathrm{~s}$, and $72^{\circ} \mathrm{C}$ for $30 \mathrm{~s}$. Relative transcript levels (mRNA abundance) were quantified according to the previously described method (Livak and Schmittgen 2001).

Statistical analysis. At least three replicates were performed for each determination, and the experiment itself was repeated three times independently. All data were subjected to one-way analysis of variance to test various treatment effects and analyzed with SPSS 20.0 statistical software package (SPSS, Chicago, IL, U.S.A.). For multiple mean comparisons, we used Tukey's least significant difference test at $5 \%$ level of significance.

\section{RESULTS}

Melatonin improves mycorrhizal colonization and enhances resistance to Fusarium oxysporum. After 35 days of AMF inoculation, the mycorrhizal colonization rate was $48.9 \%$, which was remarkably increased by $39.45 \%$ with exogenous 
melatonin application (Fig. 1A). However, the inoculation with Fusarium oxysporum (FO) significantly decreased the AMF colonization rate. The AMF colonization rate was decreased by 29.9\% following Fusarium inoculation; however, melatonin application on $\mathrm{AMF}+\mathrm{FO}$-inoculated seedlings significantly increased the colonization rate by $45.3 \%$ compared with only AMF+FO (Fig. 1A). Dry weight analysis of cucumber seedlings showed that AMF colonization increased plant biomass by $26.8 \%$, which was further significantly improved by exogenous melatonin application, although only melatonin treatment had no effect on plant biomass (Fig. 1B). However, Fusarium inoculation drastically decreased the dry weight by $48.9 \%$ compared with the control. Either AMF

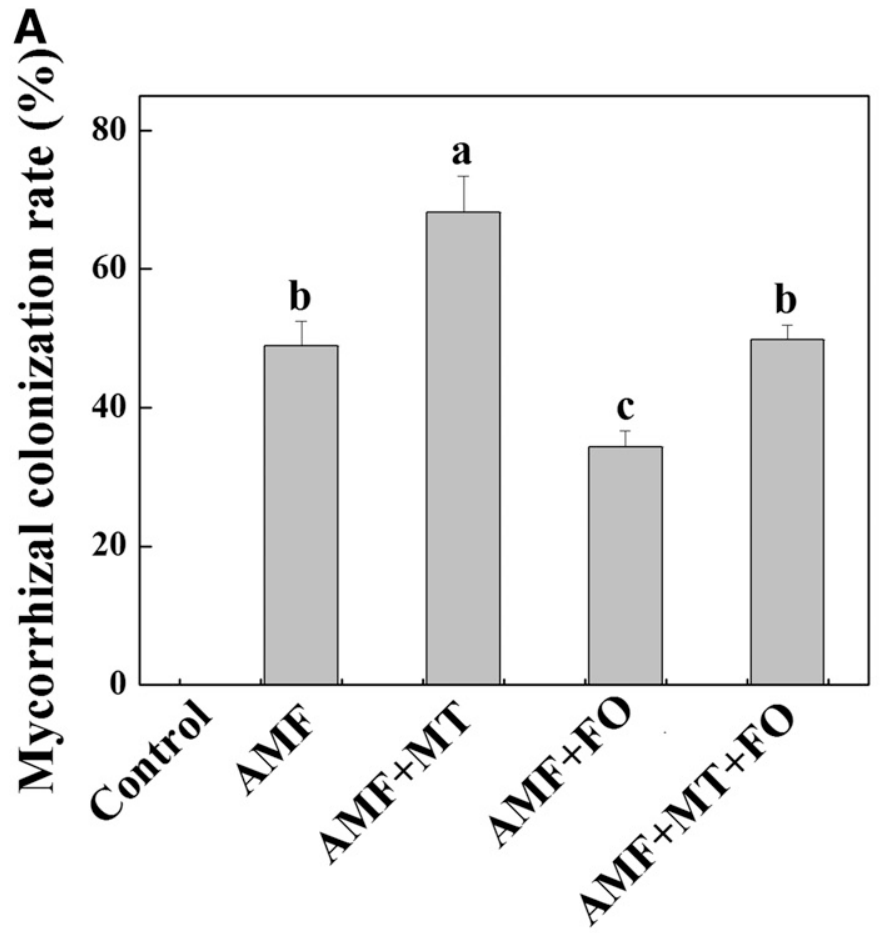

C

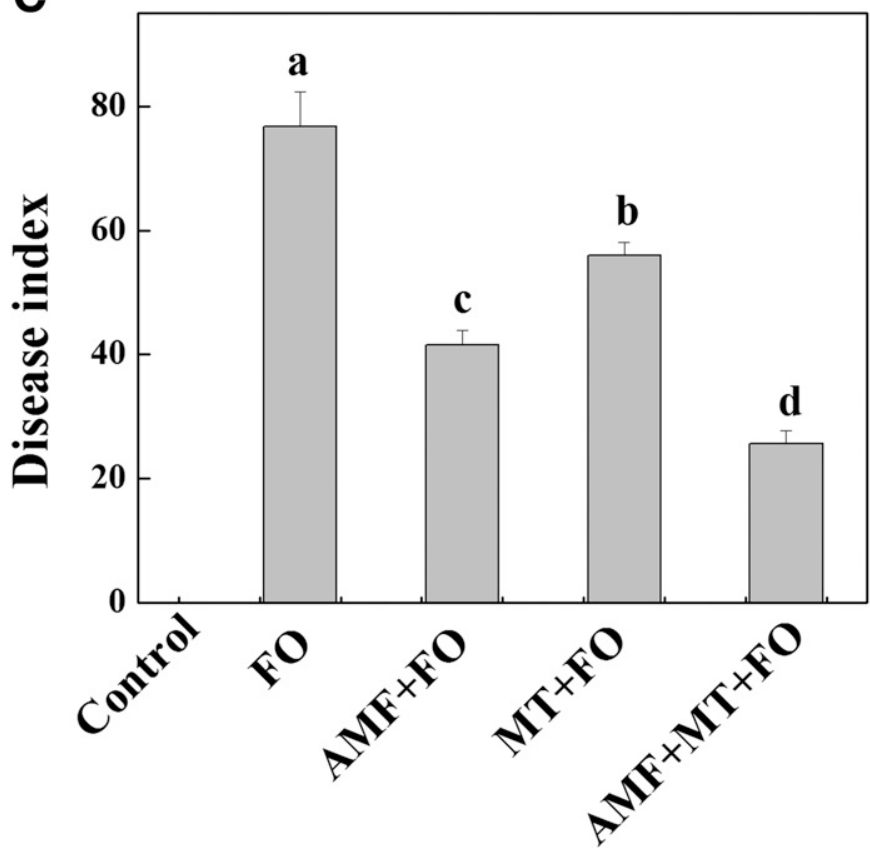

colonization or exogenous melatonin treatment, both caused a significant increase in plant biomass in Fusarium-inoculated cucumber plants; however, the combined treatment of AMF and melatonin showed the greatest effect, which increased the dry weight by $65.3 \%$ compared with the dry weight of only Fusariuminoculated plants. Comparison of disease index revealed that disease incidence was significantly decreased by either AMF or melatonin; however, the combined treatment of melatonin and AMF showed the highest effect. Consistent with the effect of AMF and melatonin on disease index, the control effect (\%) significantly increased under combined treatment of melatonin and AMF followed by sole treatment of AMF and melatonin. The control

B
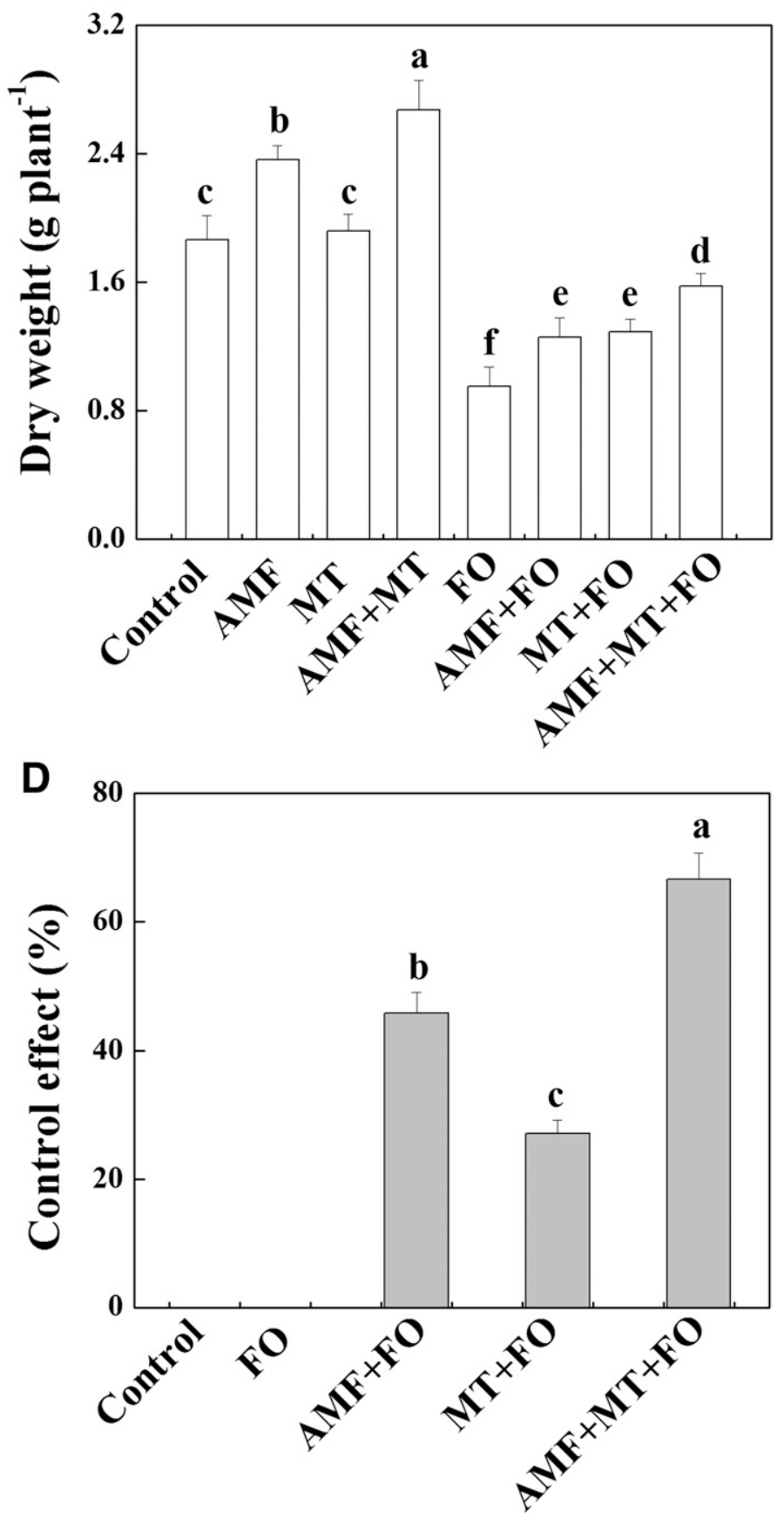

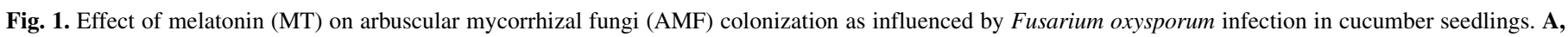

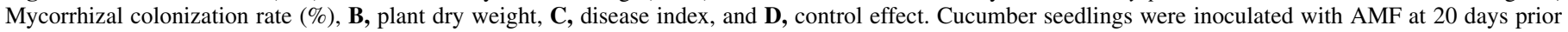

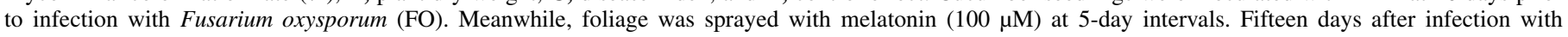

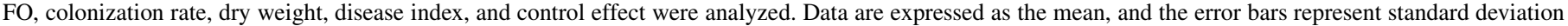
$(n=6)$. Different lowercase letters on the histograms indicate the statistically significant differences at $P<0.05$ according to Tukey's test. 
effect (\%) was 45.5 and $146.2 \%$ higher in the combined treatment of melatonin and AMF than that in the sole treatment of AMF and melatonin, respectively.

Melatonin mitigates Fusarium-induced reduction in photosynthesis. Analysis of leaf gas exchange parameters showed that inoculation with Fusarium significantly decreased the Pn, Gs, $\mathrm{Ci}$, and $\mathrm{Tr}$ by $38.5,40.9,24.7$, and $36.6 \%$, respectively, suggesting that the reduction in stomatal conductance in response to Fusarium inoculation might play a role in the attenuated $\mathrm{CO}_{2}$ assimilation and transpiration (Fig. 2). Although individual treatment with either melatonin or AMF did not affect the photosynthesis rate, their combined treatment significantly improved the rate of $\mathrm{CO}_{2}$ assimilation in absence of Fusarium. Meanwhile, either AMF inoculation or melatonin application on Fusarium-inoculated plants significantly enhanced the $\mathrm{Pn}, \mathrm{Gs}, \mathrm{Ci}$, and $\mathrm{Tr}$ compared with only Fusarium inoculation. Moreover, combined treatment of AMF and melatonin on Fusarium-inoculated plants further improved the $\mathrm{Pn}, \mathrm{Gs}$, and Tr, suggesting an additive role of AMF and melatonin in promoting leaf gas exchange under biotic stress induced by Fusarium inoculation in cucumber plants (Fig. 2).

Melatonin alleviates Fusarium-induced oxidative stress by stimulating the activity of antioxidant enzymes. Fusarium inoculation remarkably aggravated cell death as evidenced by intense red fluorescence in the root tip cells (Fig. 3A). However, cell damage greatly decreased when AMF and/or melatonin were applied alongside Fusarium inoculation, suggesting that either AMF or melatonin could protect cucumber roots from Fusarium-induced damage and their combined treatment showed a
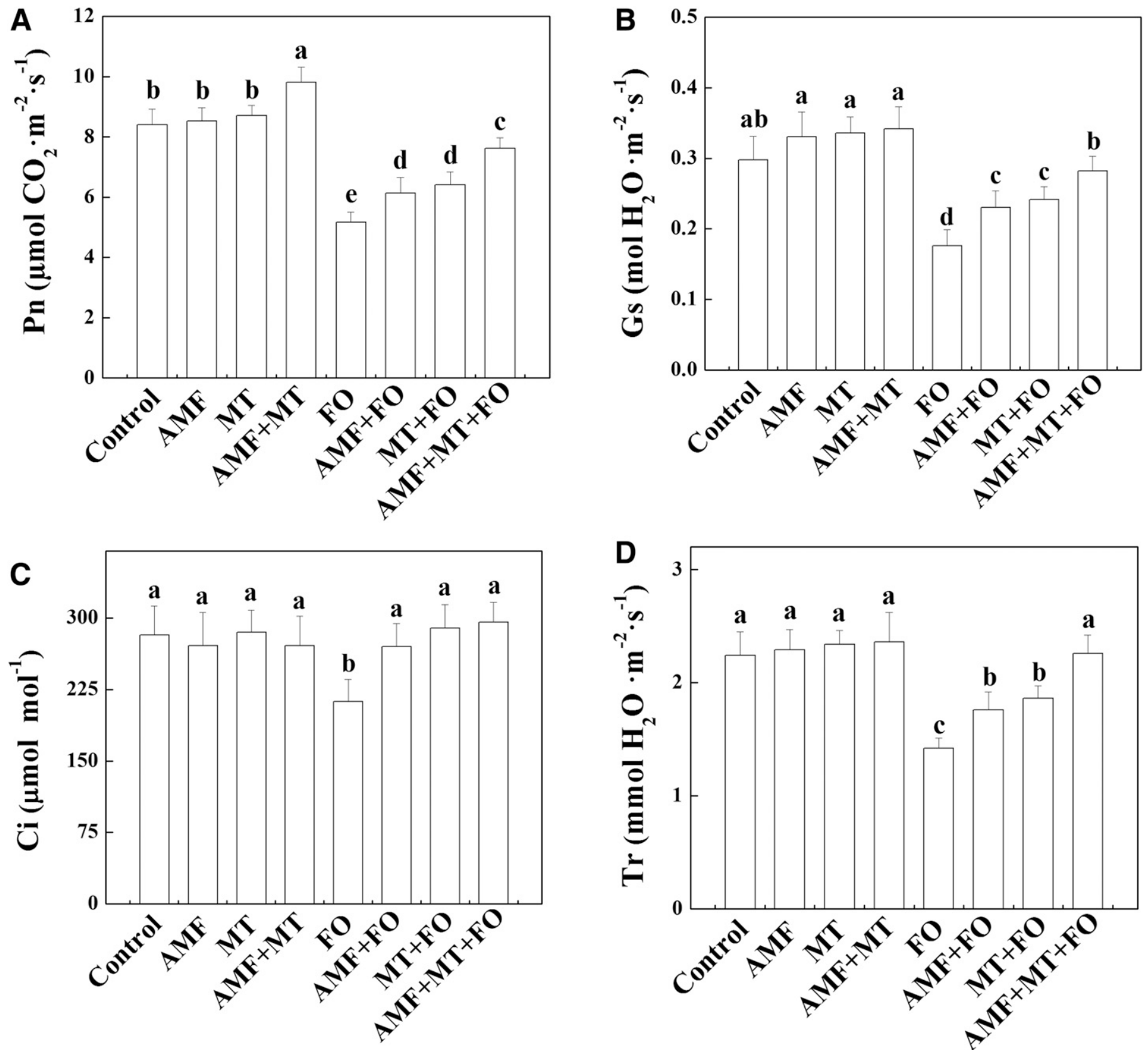

Fig. 2. Effect of melatonin (MT) and arbuscular mycorrhizal fungi (AMF) on leaf gas exchange as affected by Fusarium oxysporum infection in cucumber seedlings. A, Net photosynthesis rate $(\mathrm{Pn})$, B, stomatal conductance $(\mathrm{Gs}), \mathbf{C}$, the intercellular $\mathrm{CO}_{2}$ concentration $(\mathrm{Ci})$, and $\mathbf{D}$, the transpiration rate $(\mathrm{Tr})$. Cucumber seedlings were inoculated with AMF at 20 days prior to infection with Fusarium oxysporum (FO). Meanwhile, leaves were sprayed with melatonin (100 $\mu \mathrm{M})$ at 5day intervals. Fifteen days after infection with Fusarium oxysporum, leaf gas exchange parameters were analyzed. Data are expressed as the mean of six replicates (error bars represent standard deviation). Different lowercase letters on the histograms indicate the statistically significant differences at $P<0.05$ according to Tukey's test. 
better effect. In agreement with the root cell viability, the root activity decreased by $63.2 \%$ following Fusarium infection; however, AMF inoculation or melatonin application and their combined treatment on Fusarium-infected plants significantly increased the root activity by $70.9,82.1$, and $138.4 \%$, respectively compared with only Fusarium infection (Fig. 3B). To unveil potential mechanisms of Fusarium-induced damage, we visualized $\mathrm{H}_{2} \mathrm{O}_{2}$ accumulation by DAB staining and analyzed the levels of $\mathrm{H}_{2} \mathrm{O}_{2}$, electrolyte leakage, and lipid peroxidation in cucumber leaves. Fusarium infection remarkably increased $\mathrm{H}_{2} \mathrm{O}_{2}$ accumulation as evidenced by deep brown color deposits in DAB-stained leaves (Fig. 4A). In addition, Fusarium inoculation significantly increased the $\mathrm{H}_{2} \mathrm{O}_{2}$ content,

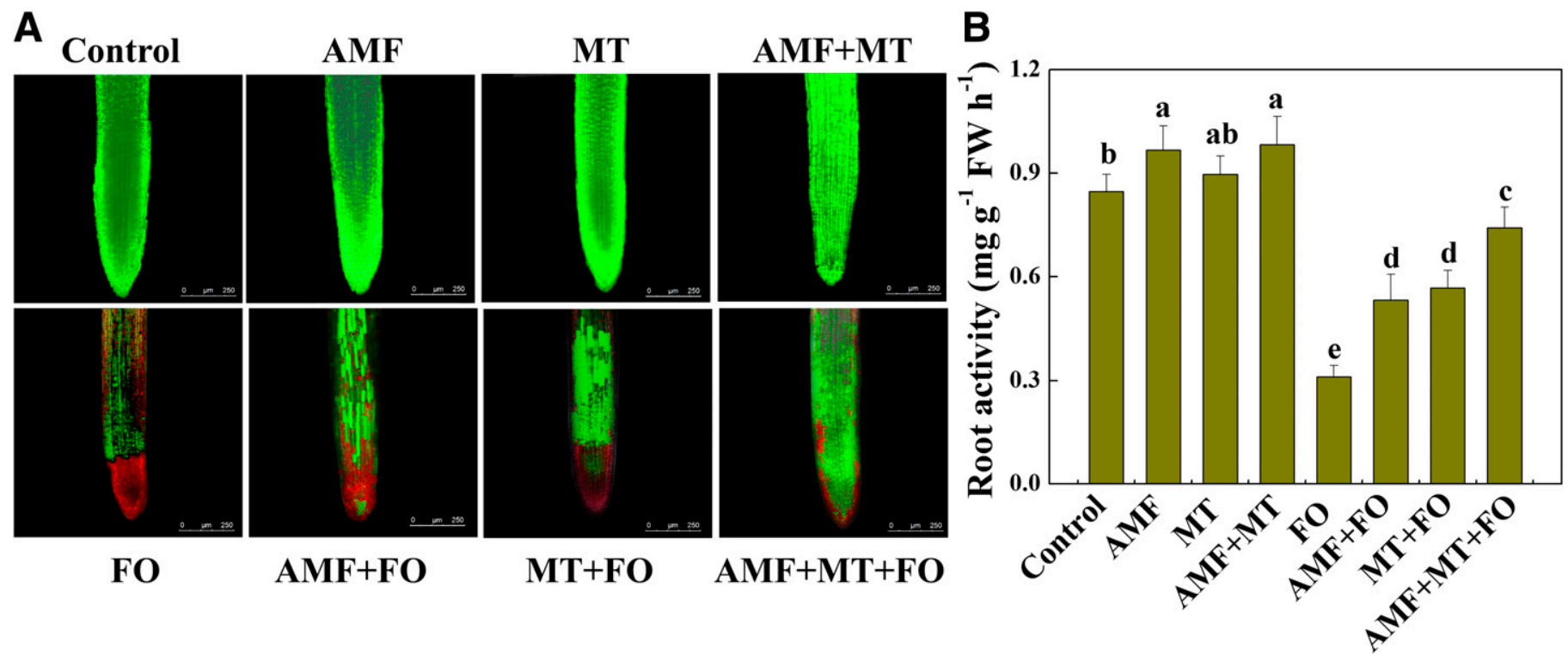

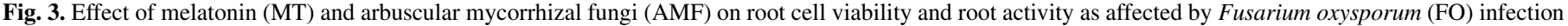

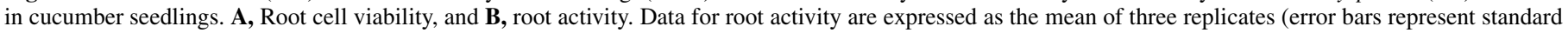
deviation). Different lowercase letters on the histograms indicate the statistically significant differences at $P<0.05$ according to Tukey's test.

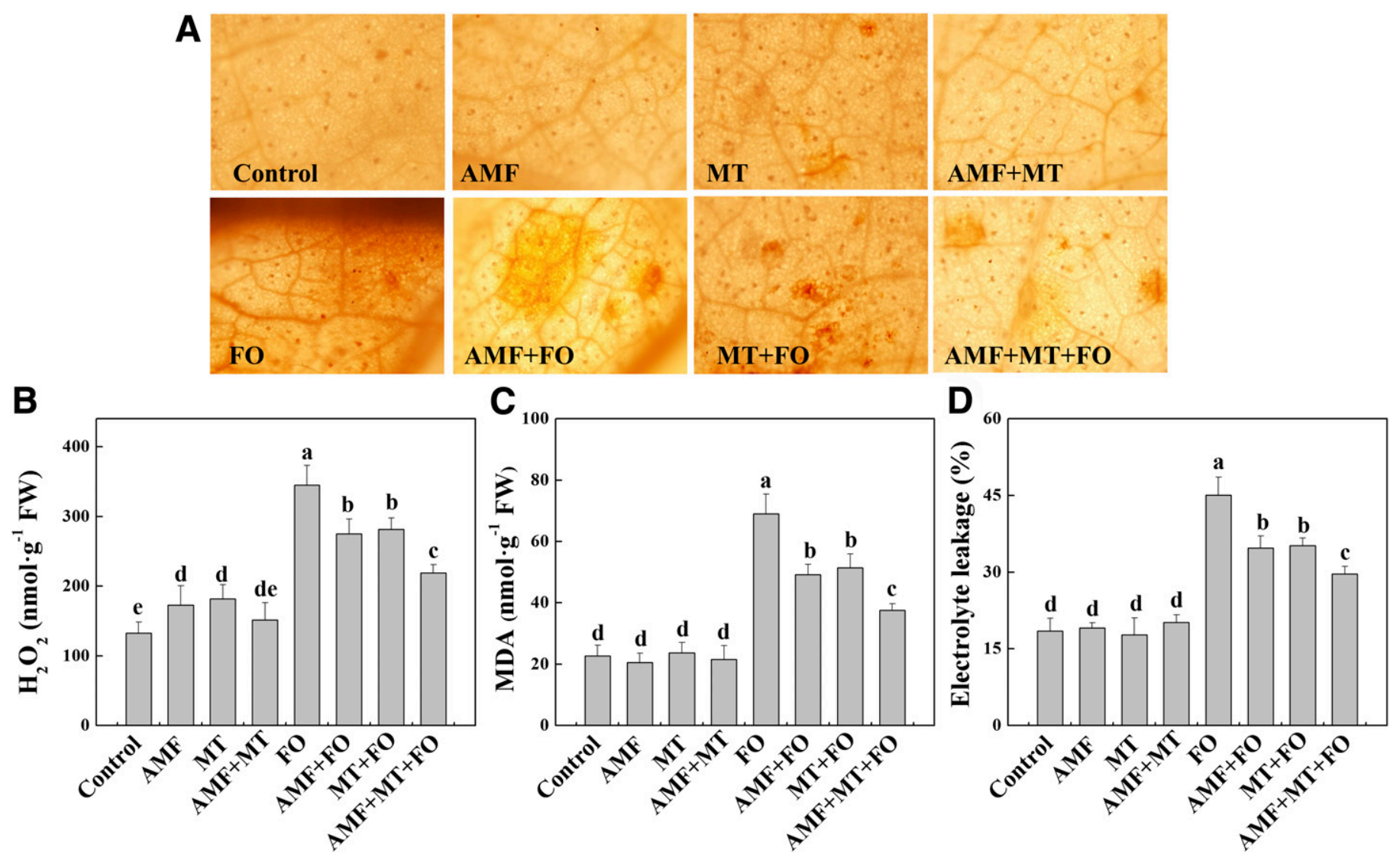

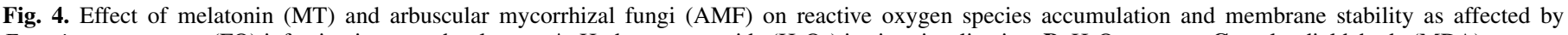

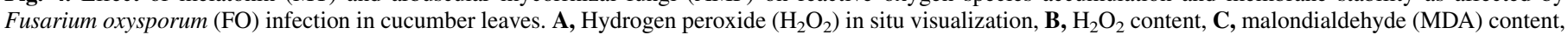

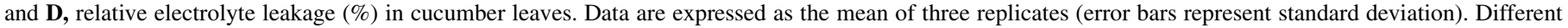
lowercase letters on the histograms indicate the statistically significant differences at $P<0.05$ according to Tukey's test. 
MDA content, and electrolyte leakage (\%) by 160.1, 204.9, and $144.4 \%$, respectively (Fig. 4B, C, and D). However, either melatonin application or AMF inoculation individually decreased the $\mathrm{H}_{2} \mathrm{O}_{2}$ accumulation, MDA content, and relative electrolyte leakage of Fusarium-stressed plants. Importantly, combined treatment with AMF and melatonin caused a further decrease in the levels of $\mathrm{H}_{2} \mathrm{O}_{2}$, MDA, and electrolyte leakage (\%), which were significantly lower than that of only AMF or melatonin treatment in Fusariuminoculated plants. Although $\mathrm{H}_{2} \mathrm{O}_{2}$ content was slightly induced by either AMF or melatonin application in absence of Fusarium, MDA content was not altered, suggesting that $\mathrm{H}_{2} \mathrm{O}_{2}$ might function as a signaling molecule rather than an inducer of oxidative stress (Fig. 4C). We then compared the activity and transcripts of some key antioxidant enzymes under different treatments (Figs. 5 and 6). The results showed that Fusarium inoculation drastically decreased the activity of SOD and APX in cucumber leaves by 41.1 and $36.9 \%$, respectively, compared with the control. However, the activity of POD and CAT was significantly elevated by 58.8 and $270.9 \%$, respectively, with Fusarium inoculation. Both AMF and melatonin applications individually increased the activity of SOD, CAT, POD, and APX in Fusarium-inoculated plants; however, their combined treatment resulted in a further promotion in the activity of antioxidative enzymes. Consistent with the activity of antioxidant enzymes, the expression of $F e-S O D$ and $c A P X$ was suppressed by Fusarium inoculation, whereas the expression of $P O D$ and $C A T$ was upregulated by Fusarium (Fig. 6). Importantly, either AMF colonization or exogenous melatonin application, both upregulated the transcript levels of $F e-S O D, P O D, C A T$, and $c A P X$, and their combined treatment showed a greater effect on those transcripts in Fusarium-infected plants, suggesting that AMF inoculation and melatonin application functioned synergistically to enhance the antioxidant enzyme activity in response to Fusarium infection.

Melatonin increases the accumulation of antioxidants and secondary metabolites under biotic stress caused by Fusarium oxysporum. To assess the role of nonenzymatic antioxidants, we analyzed the content of GSH and proline (Fig. 7). Although AMF and/or melatonin treatments significantly increased the content of GSH in absence of Fusarium infection,
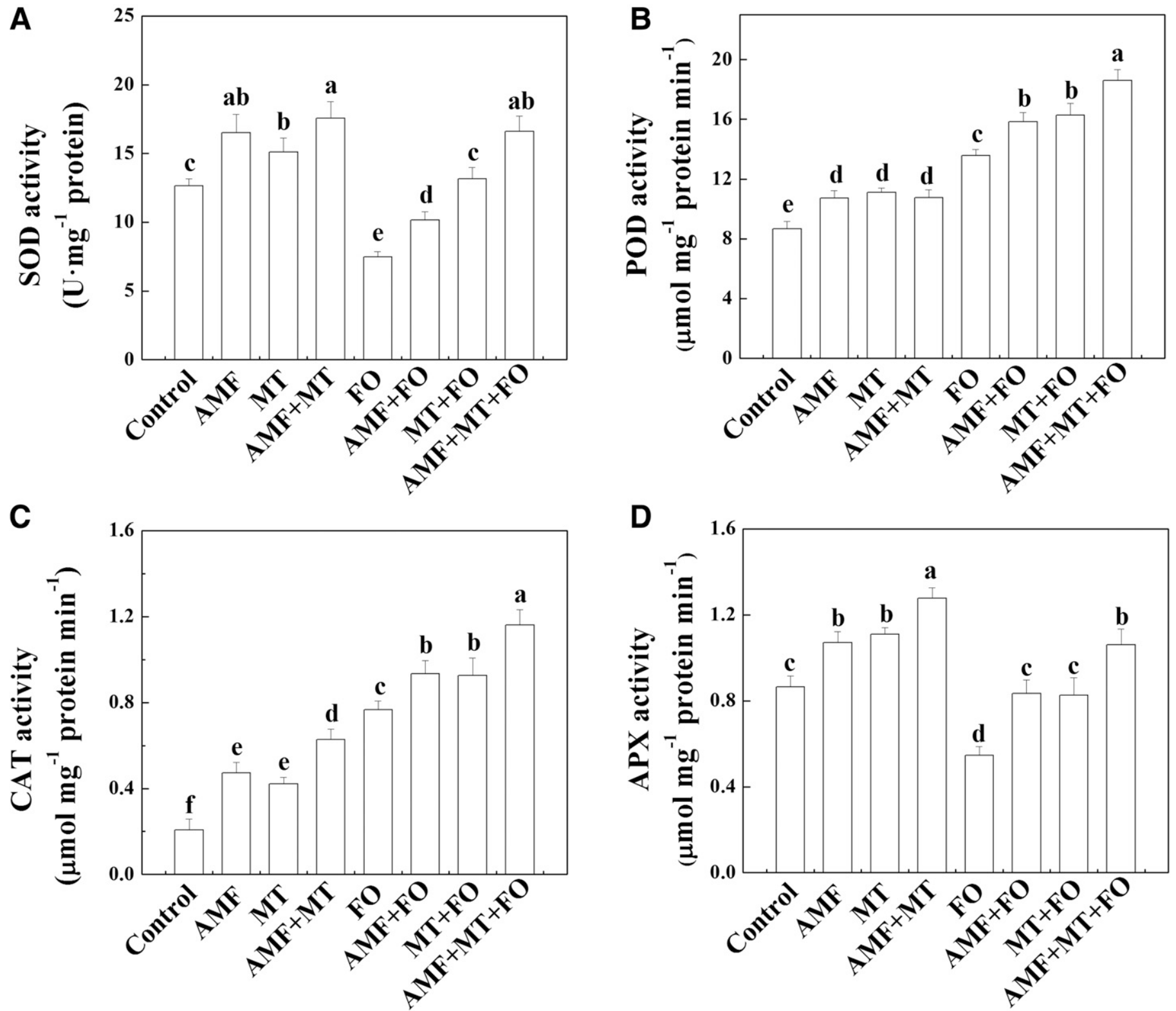

Fig. 5. Effect of melatonin (MT) and arbuscular mycorrhizal fungi (AMF) on the activity of antioxidant enzymes as influenced by Fusarium oxysporum (FO) infection in cucumber seedlings. The activity of A, superoxide dismutase (SOD), B, peroxidase (POD), $\mathbf{C}$, catalase (CAT), and D, ascorbate peroxidase (APX) in leaves. Data are expressed as the mean of three replicates (error bars represent standard deviation). Different lowercase letters on the histograms indicate the statistically significant differences at $P<0.05$ according to Tukey's test. 
none of those treatments affected proline content in a condition without Fusarium. Compared with the control, Fusarium inoculation significantly reduced the concentrations of GSH and proline by 31.9 and 37.4\%, respectively (Fig. 7A-B). However, AMF inoculation or foliar application of melatonin on Fusarium-inoculated plants increased the GSH content, and their combined treatment showed an additive effect on GSH content, causing a $96.7 \%$ increase compared with the GSH content in only Fusariuminoculated plants. Meanwhile, AMF inoculation and/or melatonin application on Fusarium-infected plants drastically increased the proline content by 110.4 to $120.7 \%$ compared with the Fusariuminoculated cucumber plants, suggesting that combined treatment of $\mathrm{AMF}$ and proline did not show any additive effect on proline content under biotic stress with Fusarium oxysporum (Fig. 7B). We also
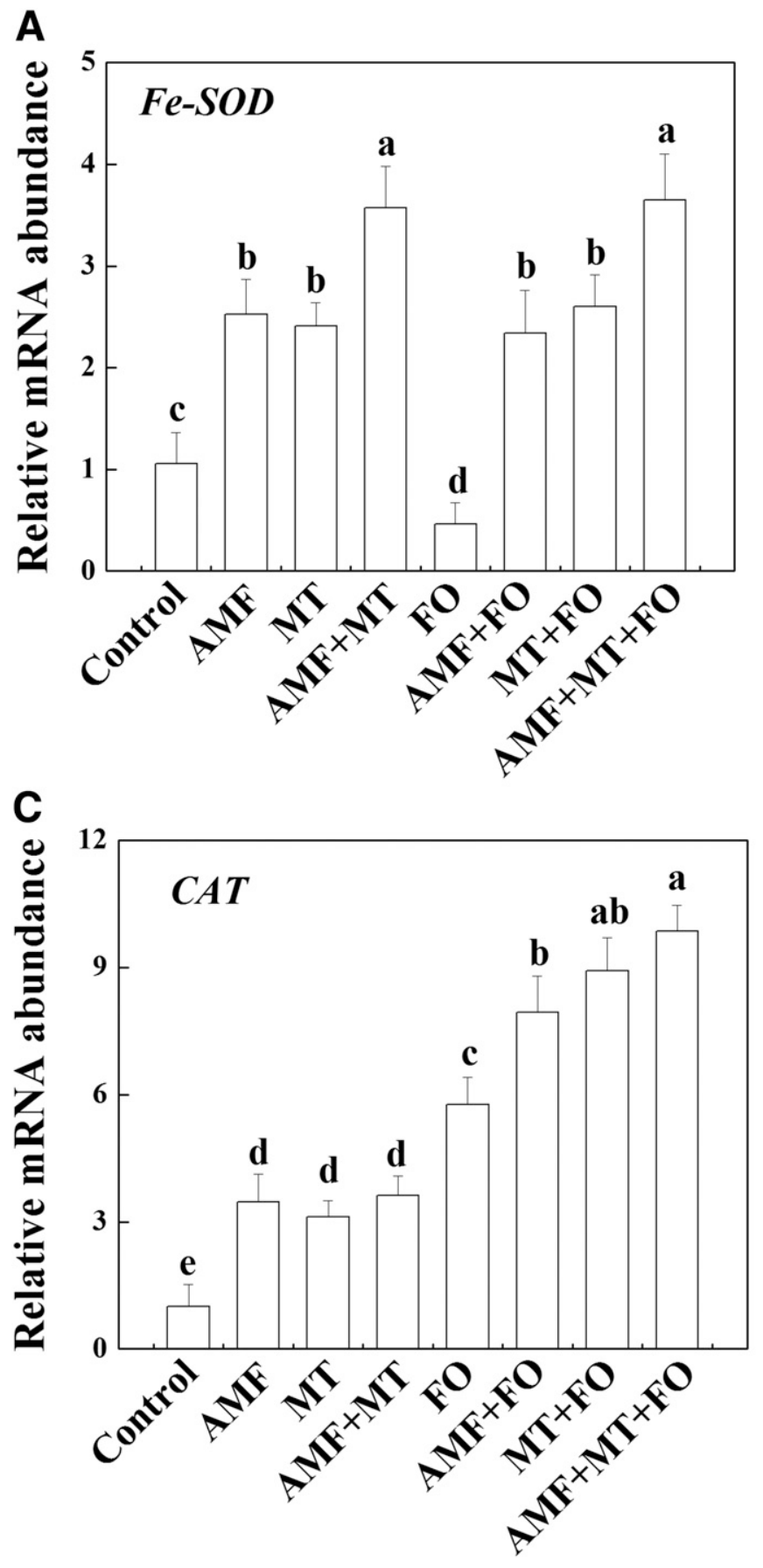

analyzed the phenols and flavonoid concentrations in cucumber leaves and found that Fusarium inoculation significantly increased their levels by 87.5 and $96.7 \%$, respectively (Fig. 7C to D). Consistent with the GSH content, AMF or melatonin treatment significantly increased the phenol and flavonoid content, and the combined treatment of AMF and melatonin caused a further increase, suggesting an additive effect of AMF and melatonin on secondary metabolism under the Fusarium stress in cucumber plants.

\section{DISCUSSION}

The occurrence of both melatonin and AMF is universal across the plant kingdom. While melatonin occurs endogenously in plant
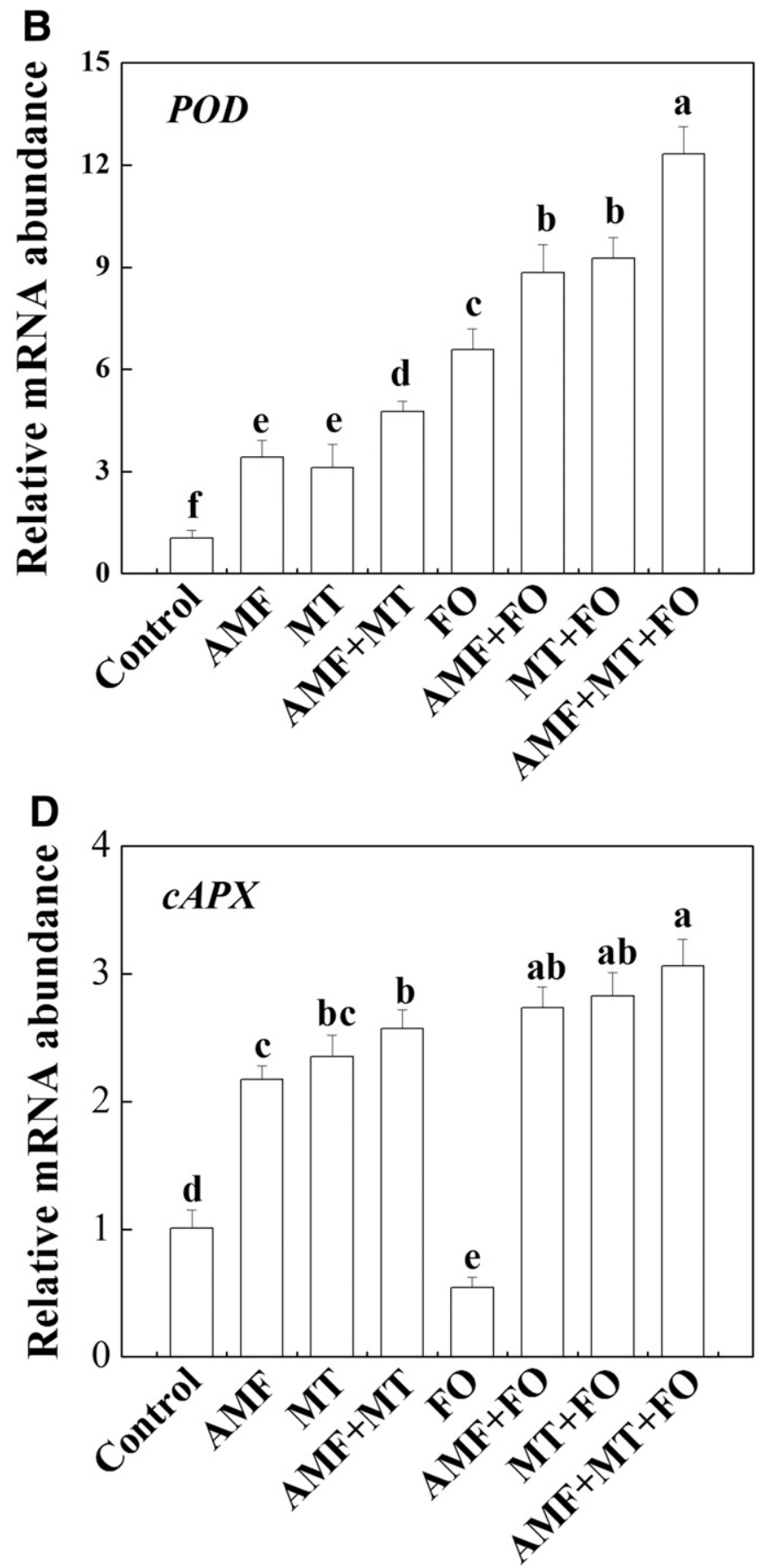

Fig. 6. Effect of melatonin (MT) and arbuscular mycorrhizal fungi (AMF) on the transcript levels of key genes encoding antioxidative enzymes as influenced by Fusarium oxysporum (FO) infection in cucumber seedlings. Relative expression levels of A, Fe-superoxide dismutase (Fe-SOD), B, peroxidase (POD), C, catalase $(C A T)$, and $\mathbf{D}$, ascorbate peroxidase $(c A P X)$ in cucumber leaves. Data are expressed as the mean of three replicates (error bars represent standard deviation). Different lowercase letters on the histograms indicate the statistically significant differences at $P<0.05$ according to Tukey's test. 
tissues (Arnao and Hernandez-Ruiz 2018), AMF colonize plant roots and provide physiological protections, including enhanced resistance to multiple plant pathogens (Miozzi et al. 2019). However, the effect of melatonin on AMF-enhanced resistance to fungal pathogens remained elusive before this study. Here we found that melatonin positively influences AMF colonization, leading to enhanced resistance to Fusarium oxysporum, a devastating plant pathogen that causes significant losses in cucumber production worldwide (Zhang et al. 2016). We also found that melatonin and/or AMF could alleviate Fusarium-caused oxidative stress by improving the antioxidative defense and secondary metabolism, which possibly facilitated efficient $\mathrm{CO}_{2}$ assimilation and biomass accumulation in cucumber plants under Fusarium oxysporum infection.

Fusarium oxysporum f. sp. cucumerinum typically infects cucumber seedlings via roots and stems; nonetheless, it prefers wounded roots for easy penetration to root tissues (Gordon 2017;
Zhang et al. 2016). Upon entry, the pathogen spreads quickly via vascular system, which eventually interrupts or totally blocks water and nutrient uptake, leading to wilting of aerial plant parts even in the abundance of water in rhizospheric soils (Gordon 2017; Wang et al. 2015). Moreover, plant pathogenic fungi significantly inhibit the photosynthetic process in plants (Li et al. 2019c; Sun et al. 2019). In the current study, Fusarium inoculation also drastically reduced the Pn and Tr, along with a significant reduction in Gs, suggesting that stomatal limitations were one of the causes of Fusarium-induced reduction in photosynthesis. These results are in agreement with a previous study in cucumber, in which infection with Fusarium oxysporum f. sp. cucumerinum significantly decreased Gs and Tr, and increased the damage to the leaf cell membranes (Wang et al. 2015). In this study, we also found significantly increased levels of $\mathrm{H}_{2} \mathrm{O}_{2}, \mathrm{MDA}$, and electrolyte leakage in Fusarium-infected plants, suggesting potential injury to
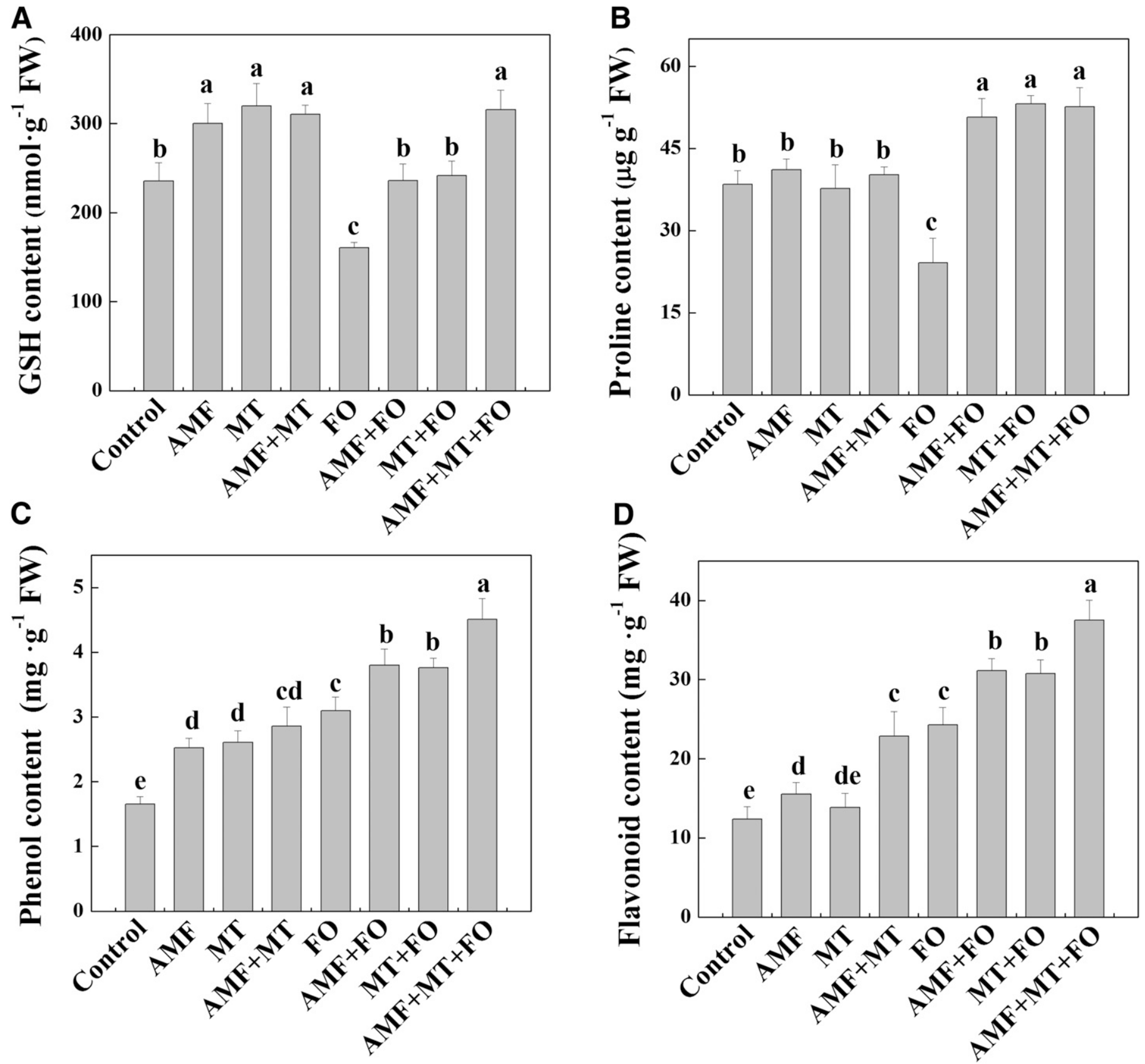

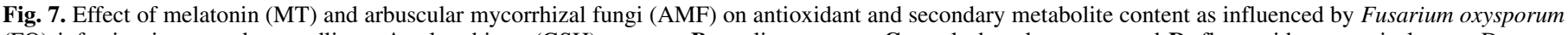

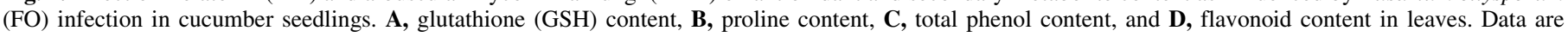

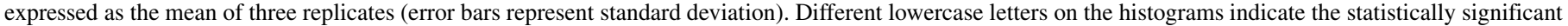
differences at $P<0.05$ according to Tukey's test. 
leaf cell membranes and nonstomatal water loss (Wang et al. 2015). However, AMF and melatonin treatment improved the $\mathrm{CO}_{2}$ assimilation rate possibly by improving Gs. Notably, melatonin can improve the activity of Calvin cycle enzymes, which may lead to an increase in photosynthetic carbon assimilation (Ding et al. 2017). In this study, consistent with the role of melatonin in ROS scavenging, the increased activity and transcript levels of antioxidant enzymes upon combined treatment of melatonin and AMF remarkably minimized the levels of $\mathrm{H}_{2} \mathrm{O}_{2}$, MDA, and electrolyte leakage, suggesting that melatonin and AMF mitigated the oxidative stress caused by Fusarium oxysporum (Sharma and Zheng 2019). These results are in accord with a recent report in cucumber, in which exogenous melatonin application increased antioxidant enzyme activity and decreased MDA content under biotic stress caused by Pseudoperonospora cubensis (Sun et al. 2019).

Melatonin functions as an antioxidant and it can reduce oxidative stress by enhancing ROS scavenging (Kanwar et al. 2018). In vitro assays show that melatonin can inhibit the growth of different fungal pathogens, such as Fusarium spp., Phytophthora spp., Alternaria spp., and Botrytis spp., suggesting its antifungal activity (Arnao and Hernandez-Ruiz 2018; Hernández-Ruiz and Arnao 2018; Mandal et al. 2018). Melatonin enhances plant resistance to fungal pathogens by inhibiting pathogen expansion, enhancing antioxidant defense and thus minimizing damage (Hernández-Ruiz and Arnao 2018). Moreover, melatonin improves resistance to Fusarium wilt in banana plants by increasing the expression of heatshock protein 90s (Wei et al. 2017). Notably, AMF inoculation can also suppress fungal diseases in many plant species (Aseel et al. 2019; Oyewole et al. 2017). Consistent with this, in the present study, exogenous melatonin application or AMF inoculation remarkably increased the resistance of cucumber plants to Fusarium wilt as evidenced by decreased disease index and increased control effect. Notably, melatonin biosynthesis also occurs in endophytic bacteria and beneficial soil fungi Trichoderma spp., which may function in host-microbe interaction as well as in resistance to plant pathogens (Arnao and Hernandez-Ruiz 2018). Therefore it is logical to speculate that exogenous melatonin might act as an inducer of plant resistance and function additively with AMF to increase resistance to Fusarium wilt in cucumber plants.

Plants produce a number of metabolites through secondary metabolic pathways, which function mostly in plant defense responses to biotic and abiotic stresses. Flavonoids and phenols are the major groups of secondary metabolites that accumulate in response to pathogen attacks. Here we found that the application of melatonin and/or inoculation with AMF increased the content of phenols and flavonoids, which might enhance defense against Fusarium wilt in cucumber. Previous studies revealed that AMF colonization could increase the accumulation of phenols and flavonoids in leaves (Chen et al. 2013). Moreover, proteomic analysis revealed that melatonin could also influence flavonoid biosynthesis (Arnao and Hernandez-Ruiz 2018). Since combined treatment of melatonin and AMF resulted in a greater accumulation of flavonoids than their sole treatment, it is highly possible that AMF and melatonin additively stimulated secondary metabolism in cucumber plants, leading to enhanced resistance to Fusarium wilt.

To sum up, we found that melatonin application increased the AMF colonization rate in cucumber roots and enhanced the control effect of AMF against Fusarium oxysporum. In addition, both melatonin and AMF promoted the photosynthetic capacity, antioxidant defense and secondary metabolite content in cucumber plants, which potentially functioned to enhance resistance against Fusarium wilt (Fig. 8). Importantly, combined treatment of melatonin and AMF showed an increased disease suppression compared with their sole treatment, suggesting that melatonin improves the AMF-enhanced plant defense against Fusarium oxysporum in cucumber. Since melatonin is considered a naturally

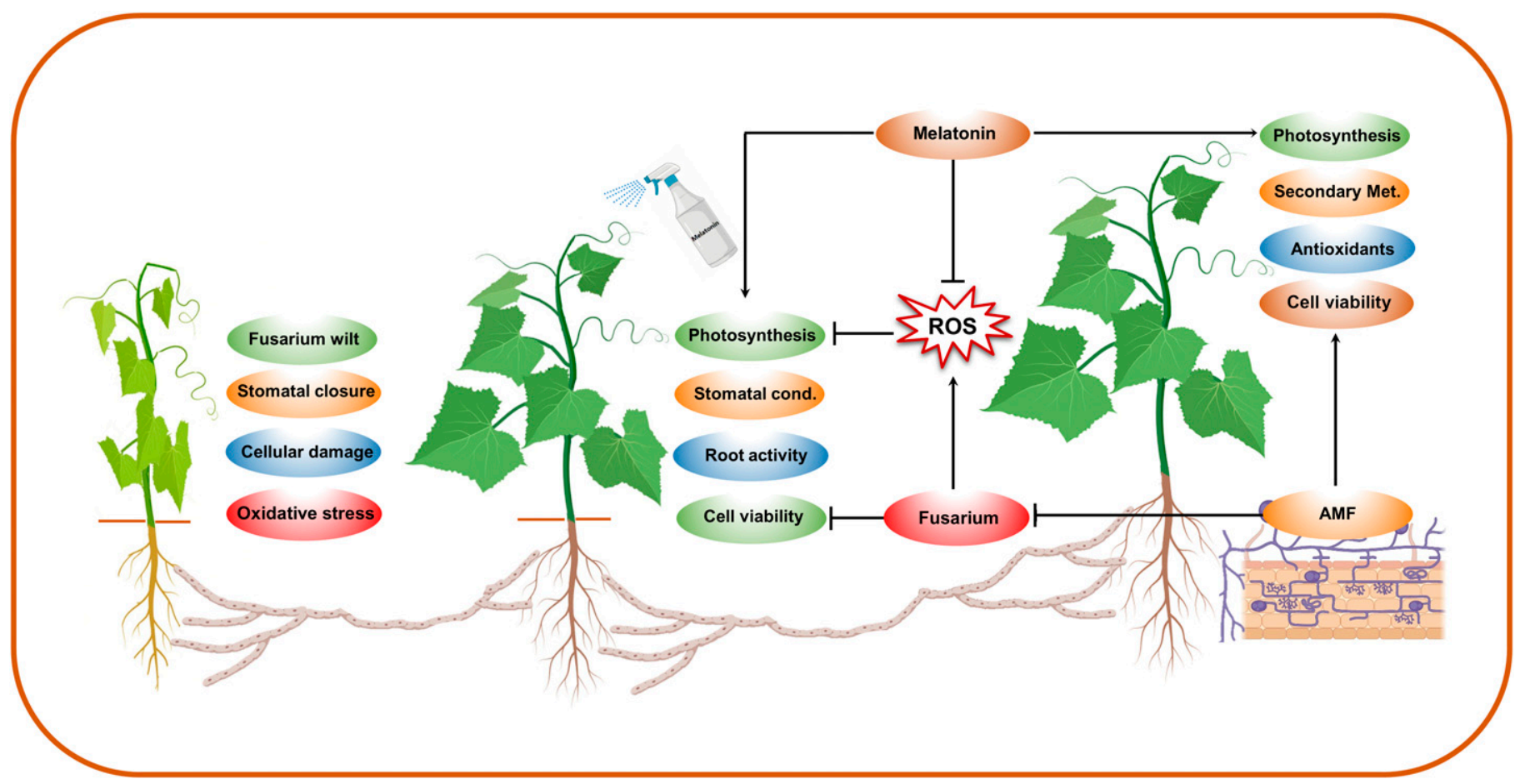

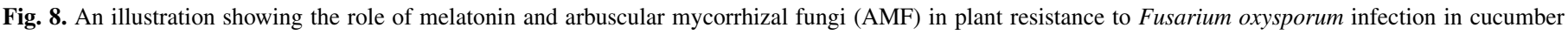

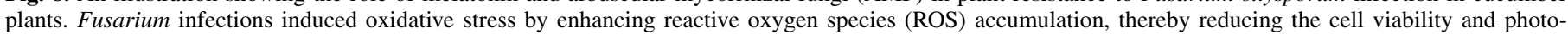

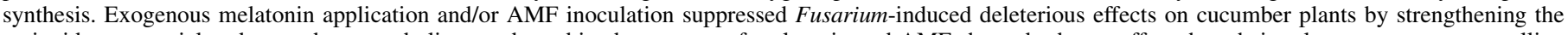

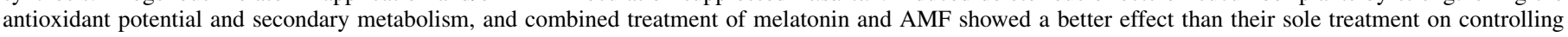

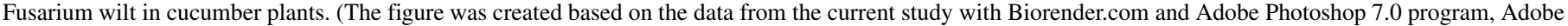
Systems, U.S.A.) 
occurring safe compound, the combined application of melatonin and AMF could be an eco-friendly strategy for the effective management of wilt disease in cucurbits.

\section{LITERATURE CITED}

Ahammed, G. J., Wu, M., Wang, Y., Yan, Y., Mao, Q., Ren, J., Ma, R., Liu, A., and Chen, S. 2020. Melatonin alleviates iron stress by improving iron homeostasis, antioxidant defense and secondary metabolism in cucumber. Sci. Hortic. (Amsterdam) 265:109205.

Ahammed, G. J., Xu, W., Liu, A., and Chen, S. 2018. COMT1 Silencing aggravates heat stress-induced reduction in photosynthesis by decreasing chlorophyll content, photosystem II activity, and electron transport efficiency in tomato. Front. Plant Sci. 9:998.

Ahammed, G. J., Xu, W., Liu, A., and Chen, S. 2019. Endogenous melatonin deficiency aggravates high temperature-induced oxidative stress in Solanum lycopersicum L. Environ. Exp. Bot. 161:303-311.

Arnao, M. B., and Hernandez-Ruiz, J. 2018. Melatonin and its relationship to plant hormones. Ann. Bot. 121:195-207.

Aseel, D. G., Rashad, Y. M., and Hammad, S. M. 2019. Arbuscular mycorrhizal fungi trigger transcriptional expression of flavonoid and chlorogenic acid biosynthetic pathways genes in tomato against tomato mosaic virus. Sci. Rep. 9:9692.

Bahadur, A., Batool, A., Nasir, F., Jiang, S., Mingsen, Q., Zhang, Q., Pan, J., Liu, Y., and Feng, H. 2019. Mechanistic insights into arbuscular mycorrhizal fungi-mediated drought stress tolerance in plants. Int. J. Mol. Sci. 20: 4199.

Bradford, M. M. 1976. A rapid and sensitive method for the quantitation of microgram quantities of protein utilizing the principle of protein-dye binding. Anal. Biochem. 72:248-254.

Cakmak, I., and Marschner, H. 1992. Magnesium deficiency and high light intensity enhance activities of superoxide dismutase, ascorbate peroxidase, and glutathione reductase in bean leaves. Plant Physiol. 98:1222-1227.

Chen, S., Jin, W., Liu, A., Zhang, S., Liu, D., Wang, F., Lin, X., and He, C. 2013. Arbuscular mycorrhizal fungi (AMF) increase growth and secondary metabolism in cucumber subjected to low temperature stress. Sci. Hortic. (Amsterdam) 160:222-229.

Chen, S., Ren, J., Zhao, H., Wang, X., Wang, T., Jin, S., Li, C., Liu, A., Lin, X., and Ahammed, G. J. 2019. Trichoderma harzianum improves defense against Fusarium oxysporum by regulating ROS and RNS metabolism, redox balance and energy flow in cucumber roots. Phytopathology 109:972-982.

Chen, S., Zhao, H., Zou, C., Li, Y., Chen, Y., Wang, Z., Jiang, Y., Liu, A., Zhao, P., Wang, M., and Ahammed, G. J. 2017b. Combined inoculation with multiple arbuscular mycorrhizal fungi improves growth, nutrient uptake and photosynthesis in cucumber seedlings. Front. Microbiol. 8:2516.

Chen, S.-C., Zhao, H.-J., Wang, Z.-H., Zheng, C.-X., Zhao, P.-Y., Guan, Z.-H., Qin, H.-Y., Liu, A.-R., Lin, X.-M., and Ahammed, G.-J. 2017a. Trichoderma harzianum-induced resistance against Fusarium oxysporum involves regulation of nuclear DNA content, cell viability and cell cycle-related genes expression in cucumber roots. Eur. J. Plant Pathol. 147:43-53.

Ding, F., Liu, B., and Zhang, S. 2017. Exogenous melatonin ameliorates coldinduced damage in tomato plants. Sci. Hortic. (Amsterdam) 219:264-271.

Ding, J., Zhang, Y., Zhang, H., Li, X., Sun, Z., Liao, Y., Xia, X., Zhou, Y., Shi, K., and Yu, J. 2014. Effects of Fusarium oxysporum on rhizosphere microbial communities of two cucumber genotypes with contrasting Fusarium wilt resistance under hydroponic condition. Eur. J. Plant Pathol. 140:643-653.

Gao, P., Li, Y., Guo, Y., and Duan, T. 2018. Co-inoculation of lucerne (Medicago sativa) with an AM fungus and a rhizobium reduces occurrence of spring black stem and leaf spot caused by Phoma medicaginis. Crop Pasture Sci. 69:933-943.

Giannopolitis, C. N., and Ries, S. K. 1977. Superoxide dismutases: I. occurrence in higher plants. i. occurrence in higher plants. Plant Physiol. 59:309-314.

Giovannetti, M., and Mosse, B. 1980. An evaluation of techniques for measuring vesicular arbuscular mycorrhizal infection in roots. New Phytol. 84: 489-500.

Gordon, T. R. 2017. Fusarium oxysporum and the Fusarium wilt syndrome. Annu. Rev. Phytopathol. 55:23-39.

Hasan, M. K., Ahammed, G. J., Sun, S., Li, M., Yin, H., and Zhou, J. 2019. Melatonin inhibits cadmium translocation and enhances plant tolerance by regulating sulfur uptake and assimilation in Solanum lycopersicum L. J. Agric. Food Chem. 67:10563-10576.

Hernández-Ruiz, J., and Arnao, M. 2018. Relationship of melatonin and salicylic acid in biotic/abiotic plant stress responses. Agronomy (Basel) 8:33.

Kanwar, M. K., Yu, J., and Zhou, J. 2018. Phytomelatonin: Recent advances and future prospects. J. Pineal Res. 65:e12526.

Li, S., Xu, Y., Bi, Y., Zhang, B., Shen, S., Jiang, T., and Zheng, X. 2019a. Melatonin treatment inhibits gray mold and induces disease resistance in cherry tomato fruit during postharvest. Postharvest Biol. Technol. 157: 110962.
Li, T., Wu, Q., Zhu, H., Zhou, Y., Jiang, Y., Gao, H., and Yun, Z. 2019 b. Comparative transcriptomic and metabolic analysis reveals the effect of melatonin on delaying anthracnose incidence upon postharvest banana fruit peel. BMC Plant Biol. 19:289.

Li, X., Ahammed, G. J., Li, Z., Tang, M., Yan, P., and Han, W. 2016. Decreased biosynthesis of jasmonic acid via lipoxygenase pathway compromised caffeine-induced resistance to Colletotrichum gloeosporioides under elevated $\mathrm{CO}_{2}$ in tea seedlings. Phytopathology 106:1270-1277.

Li, X., Brestic, M., Tan, D. X., Zivcak, M., Zhu, X., Liu, S., Song, F., Reiter, R. J., and Liu, F. 2018. Melatonin alleviates low PS I-limited carbon assimilation under elevated $\mathrm{CO}_{2}$ and enhances the cold tolerance of offspring in chlorophyll b-deficient mutant wheat. J. Pineal Res. 64:e12453.

Li, Y., Duan, T., Nan, Z., and Li, Y. 2019c. Arbuscular mycorrhizal fungus alleviates alfalfa leaf spots caused by Phoma medicaginis revealed by RNAseq analysis. J. Appl. Microbiol. jam.14387.

Lin, Y., Fan, L., Xia, X., Wang, Z., Yin, Y., Cheng, Y., and Li, Z. 2019. Melatonin decreases resistance to postharvest green mold on citrus fruit by scavenging defense-related reactive oxygen species. Postharvest Biol. Technol. 153:21-30.

Liu, C., Chen, L., Zhao, R., Li, R., Zhang, S., Yu, W., Sheng, J., and Shen, L. 2019. Melatonin induces disease resistance to Botrytis cinerea in tomato fruit by activating jasmonic acid signaling pathway. J. Agric. Food Chem. 67:6116-6124.

Livak, K. J., and Schmittgen, T. D. 2001. Analysis of relative gene expression data using real-time quantitative PCR and the 2(T)(-Delta Delta C) method. Methods 25:402-408.

Mandal, M. K., Suren, H., Ward, B., Boroujerdi, A., and Kousik, C. 2018. Differential roles of melatonin in plant-host resistance and pathogen suppression in cucurbits. J. Pineal Res. 65:e12505.

Mathur, S., Sharma, M. P., and Jajoo, A. 2018. Improved photosynthetic efficacy of maize (Zea mays) plants with arbuscular mycorrhizal fungi (AMF) under high temperature stress. J. Photochem. Photobiol. B 180:149-154.

Miozzi, L., Vaira, A. M., Catoni, M., Fiorilli, V., Accotto, G. P., and Lanfranco, L. 2019. Arbuscular mycorrhizal symbiosis: Plant friend or foe in the fight against viruses? Front. Microbiol. 10:1238.

Nakano, Y., and Asada, K. 1981. Hydrogen peroxide is scavenged by ascorbatespecific peroxidase in spinach chloroplasts. Plant Cell Physiol. 22:867-880.

Oyewole, B. O., Olawuyi, O. J., Odebode, A. C., and Abiala, M. A. 2017. Influence of arbuscular mycorrhiza fungi (AMF) on drought tolerance and charcoal rot disease of cowpea. Biotechnol. Rep. (Amst.) 14:8-15.

Rahman, I., Kode, A., and Biswas, S. K. 2006. Assay for quantitative determination of glutathione and glutathione disulfide levels using enzymatic recycling method. Nat. Protoc. 1:3159-3165.

Rathod, D. P., Brestic, M., and Shao, H. B. 2011. Chlorophyll a fluorescence determines the drought resistance capabilities in two varieties of mycorrhized and non-mycorrhized Glycine max. Linn. J. Microbiol. Res. 5:4197-4206.

Sharma, A., and Zheng, B. 2019. Melatonin mediated regulation of drought stress: Physiological and molecular aspects. Plants 8:190.

Shi, Y. Z., Zhang, X. L., Su, S. X., Lan, Z. J., Li, K., Wang, Y. M., Wang, F. Y., and Chan, Y. L. 2017. Mycorrhizal relationship in lupines: A review. Legume Res. 40:965-973.

Shi, Z., Chen, Y., Hou, X., Gao, S., and Wang, F. 2013. Arbuscular mycorrhizal fungi associated with tree peony in 3 geographic locations in China. Turk. J. Agric. For. 37:726-733.

Sun, Y., Liu, Z., Lan, G., Jiao, C., and Sun, Y. 2019. Effect of exogenous melatonin on resistance of cucumber to downy mildew. Sci. Hortic. (Amsterdam) 255:231-241.

Wang, M., Sun, Y., Sun, G., Liu, X., Zhai, L., Shen, Q., and Guo, S. 2015. Water balance altered in cucumber plants infected with Fusarium oxysporum $\mathrm{f}$. sp. cucumerinum. Sci. Rep. 5:7722.

Wei, Y., Hu, W., Wang, Q., Zeng, H., Li, X., Yan, Y., Reiter, R. J., He, C., and Shi, H. 2017. Identification, transcriptional and functional analysis of heatshock protein 90s in banana (Musa acuminata L.) highlight their novel role in melatonin-mediated plant response to Fusarium wilt. J. Pineal Res. 62.

Willekens, H., Chamnongpol, S., Davey, M., Schraudner, M., Langebartels, C., Van Montagu, M., Inzé, D., and Van Camp, W. 1997. Catalase is a sink for $\mathrm{H}_{2} \mathrm{O}_{2}$ and is indispensable for stress defence in $\mathrm{C}_{3}$ plants. EMBO J. 16: 4806-4816.

Zhang, D., Meng, K. X., Hao, Y. H., Fan, H. Y., Cui, N., Wang, S. S., and Song, T. F. 2016. Comparative proteomic analysis of cucumber roots infected by Fusarium oxysporum f. sp. cucumerium Owen. Physiol. Mol. Plant Pathol. 96:77-84.

Zhang, S., Zheng, X., Reiter, R. J., Feng, S., Wang, Y., Liu, S., Jin, L., Li, Z., Datla, R., and Ren, M. 2017. Melatonin attenuates potato late blight by disrupting cell growth, stress tolerance, fungicide susceptibility and homeostasis of gene expression in Phytophthora infestans. Front. Plant Sci. 8:1993.

Zhang, Y., Liang, Y., Zhao, X., Jin, X., Hou, L., Shi, Y., and Ahammed, G. J. 2019. Silicon compensates phosphorus deficit-induced growth inhibition by improving photosynthetic capacity, antioxidant potential, and nutrient homeostasis in tomato. Agronomy (Basel) 9:733. 